\title{
Study of Lipid Profile and Carcinoembryonic Antigen in Breast Cancer Patients
}

\author{
Anjana Sharma ${ }^{1}$, Shehreen Akhtar ${ }^{2}$, Bushra Fiza ${ }^{3}$, Maheep Sinha ${ }^{4}$
}

\begin{abstract}
Aim: The purpose of this study was to evaluate the levels of carcinoembryonic antigen (CEA) and lipid profile [total cholesterol (TC), serum triglycerides (TG), high-density lipoprotein (HDL), low-density lipoprotein (LDL), and very-low-density lipoprotein (VLDL)] in patients with breast cancer and compared them with healthy individuals as a control group.

Materials and methods: In the present study, 100 women included 50 breast cancer patients with age $>18$ years and 50 controls with similar age range were assessed for CEA and lipid profile.

Result: The present study has demonstrated that the levels of CEA, TC, LDL, VLDL, and TG were significantly higher and the levels of HDL were lower in patients with breast cancer.

Conclusion: The study concluded that CEA levels were higher in the case group and it is one of the most commonly expressed biological markers in breast cancer patients and when lipid profile was compared between breast cancer patients and the control group, then, TC, LDL, VLDL, and TG were significantly higher and the levels of HDL were lower in patients with breast cancer.

Keywords: Carcinogenesis, Carcinoma, Lipids, Lipoproteins, Malignancy.

Journal of Mahatma Gandhi University of Medical Sciences \& Technology (2020): 10.5005/jp-journals-10057-0114
\end{abstract}

\section{InTRODUCTION}

Cancer is a major public health problem and is one of the main causes of death worldwide. ${ }^{1}$ It is the second leading cause of death after cardiovascular diseases (CVDs), ${ }^{2}$ affecting all categories of persons. Breast cancer is the most common malignancy and a leading cause of cancer death among women worldwide ${ }^{3}$. There are about 1 million new breast cancer patients every year in the world and the age of onset tends to be younger. ${ }^{4}$ Breast cancer is a multistep process characterized by uncontrolled cell growth in the tissue of the breast.

Early detection of cancer offers the best chance to diagnose cancer at an early stage and reduce morbidity and mortality. ${ }^{5}$ Among the various biological markers, carcinoembryonic antigen (CEA) is one of the most commonly expressed biological markers in patients with breast cancer. ${ }^{6}$ Carcinoembryonic antigen is a high molecular weight cell surface glycoprotein ${ }^{7}$ and it is the most widely used tumor marker in the clinical practice. ${ }^{8}$ Normal levels of CEA are observed in benign diseases and increased levels are found in breast cancer, hepatocellular carcinoma, colon cancer, lung cancer, etc. ${ }^{9}$

Apart from tumor markers, there is a need for simple biochemical investigations which can be easily assayed and can detect the status of malignancy. The lipid profile is among them. Researchers have reported the association of lipoproteins and lipids with different types of cancers. Alterations in the circulatory lipid level are associated with the etiology of carcinoma of breast. ${ }^{10}$ Adiposity is associated with both increased risk of glucose intolerance or diabetes, and breast cancer risk. ${ }^{11}$ Therefore, along with CEA, this study aimed to compare serum lipid profiles between breast cancer patients and healthy individuals.

\section{Materials and Methods}

The present study was conducted in the Department of Biochemistry in collaboration with the Department of Medical
1-4Department of Biochemistry, Mahatma Gandhi Medical College and
Hospital, Jaipur, Rajasthan, India
Corresponding Author:Shehreen Akhtar, Department of Biochemistry,
Mahatma Gandhi Medical College and Hospital, Jaipur, Rajasthan,
India, Phone: +91-8439574700, e-mail: Shehreen.akhtar08@gmail. com

How to cite this article: Sharma A, Akhtar S, Fiza B, et al. Study of Lipid Profile and Carcinoembryonic Antigen in Breast Cancer Patients. J Mahatma Gandhi Univ Med Sci Tech 2020;5(1):1-3.

Source of support: Nil

Conflict of interest: None

Oncology at Mahatma Gandhi Medical College and Hospital, Jaipur, Rajasthan.

One hundred women including 50 breast cancer patients with age $>18$ years and 50 controls with similar age range were included in the study and women below the age of 18 years and patients on lipid-lowering drugs, diabetic, and hypertension were excluded.

Blood samples were collected using the standard aseptic technique and analyzed for the following parameters:

- Serum CEA.

- Lipid profile [total cholesterol (TC), serum triglycerides (TG), high-density lipoprotein (HDL), low-density lipoprotein (LDL), very-low-density lipoprotein (VLDL)].

\section{Statistical Analysis}

Results obtained after analysis were presented as mean \pm SD between two groups, i.e., breast cancer patients $(n=50)$ and control group $(n=50)$. The result of the patient group was compared with those of the control group by applying the Student's $t$ test. A $p$ value of $\leq 0.05$ was considered significant for all statistical tests. 


\section{Result}

In the present study, the mean age in breast cancer cases $(54.54 \pm$ 14.21 years) and the control group $(58.26 \pm 8.53)$ was comparable. The levels of CEA (Table 1 and Fig. 1), TC, LDL, VLDL, and TG were significantly higher and the levels of HDL were lower (Table 1 and Fig. 2) in patients with breast cancer.

\section{Discussion}

Cancerous tumors in the breast usually grow slowly. So, early diagnosis is the best way to reduce the risk of dying from breast cancer. ${ }^{12}$ The present study was planned to evaluate the CEA and lipid profile level in 50 breast cancer patients and compared them with 50 healthy individuals. Patients were selected based on the predefined inclusion and exclusion criteria and after obtaining informed consent.

The mean age in breast cancer cases was $54.54 \pm 14.21$ years and in healthy controls $58.26 \pm 8.53$ years, and it was found statistically significant with a $p$ value of 0.000 . Carcinoembryonic antigen is a glycoprotein involved in cell adhesion. Elevated levels of serum CEA are related to the extent of the disease, site, and degree of differentiation of tumor. ${ }^{13}$ When CEA levels were compared between breast cancer cases and healthy controls, then a statistically significant difference in mean of CEA levels was found between them, as shown in Table 1.

In the present study, it was observed that CEA levels in breast cancer were higher than healthy controls. Similar findings were reported by Faridi et al., ${ }^{14}$ they further concluded that serum CEA is a tumor marker of breast carcinoma. Its level decreased after treatment and more decrease in levels after chemo/radiotherapy following surgery. Another study by Li et al. ${ }^{15}$ also demonstrated similar results and concluded that CEA had more significant prognostic significance for pretreatment primary breast cancer.

In the present study, when the levels of all the components of lipid profile, i.e., TC, TG, LDL, HDL, and VLDL, were compared between breast cancer patients and healthy individuals then a statistically significant increased were found in TC, TG, LDL, and VLDL levels and deceased in HDL levels in breast cancer patients, as shown in Table 1. Similar findings were reported by Bhat et al., ${ }^{16}$ they further concluded that elevated lipid levels precede the development of obesity and breast cancer and thus, may have an etiological or predictive significance.

Abdelsalam et al. ${ }^{17}$ demonstrated in their study that increased TC and LDL levels and decreased HDL levels increase the risk of coronary heart disease.

A major link has been established between the biosynthesis of cholesterol and cell growth. If no exogenous cholesterol is available and cholesterol synthesis is inhibited, cell growth will be blocked. Cholesterol inhibition, either by decreasing cholesterol availability (lowering of plasma cholesterol) or by decreasing intracellular cholesterol synthesis could inhibit tumor cell growth and possibly prevent carcinogenesis. ${ }^{16}$

\section{CONCLUSION}

The present study has demonstrated that the levels of CEA, TC, LDL, VLDL, and TG were significantly higher and the levels of HDL were lower in patients with breast cancer. Carcinoembryonic antigen is one of the most commonly expressed biological markers in

Table 1: Comparison of CEA and lipid profile levels between cases and control group

\begin{tabular}{lllll}
\hline & Cases $(n=50)$ & Controls $(n=50)$ & t value & $p$ value \\
\hline CEA $(\mathrm{ng} / \mathrm{mL})$ & $32.47 \pm 88.84$ & $1.91 \pm 0.72$ & -2.43 & 0.016 \\
Total cholesterol $(\mathrm{mg} / \mathrm{dL})$ & $227.41 \pm 33.09$ & $115.48 \pm 42.67$ & -12.65 & 0.000 \\
Serum triglycerides $(\mathrm{mg} / \mathrm{dL})$ & $172.99 \pm 21.84$ & $109.28 \pm 20.92$ & -14.21 & 0.000 \\
HDL $(\mathrm{mg} / \mathrm{dL})$ & $33.97 \pm 5.67$ & $43.49 \pm 6.13$ & 5.15 & 0.000 \\
LDL $(\mathrm{mg} / \mathrm{dL})$ & $136.45 \pm 32.29$ & $132.39 \pm 21.97$ & -4.05 & 0.001 \\
VLDL $(\mathrm{mg} / \mathrm{dL})$ & $32.09 \pm 5.28$ & $17.45 \pm 6.29$ & -11.53 & 0.000 \\
\hline
\end{tabular}

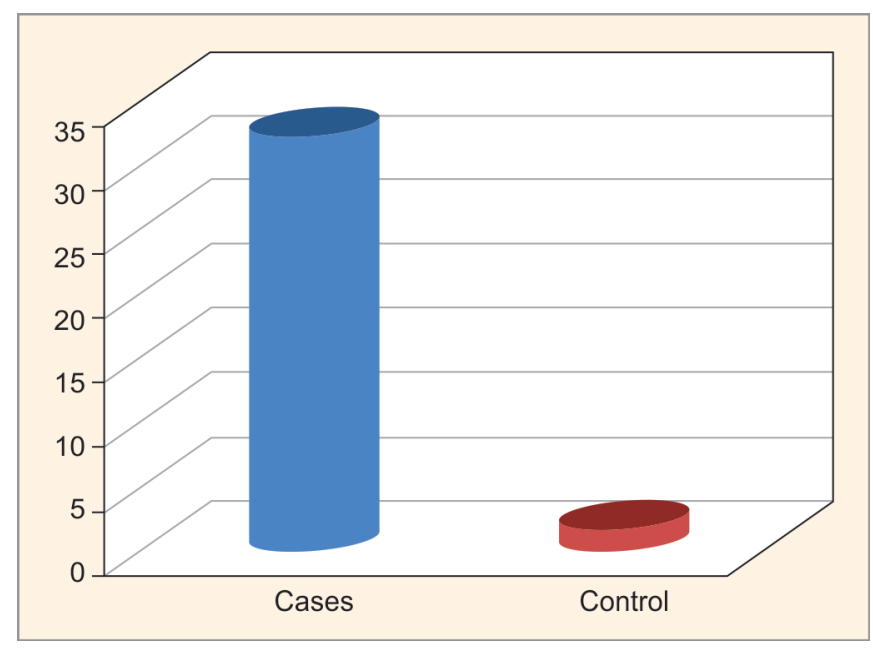

Fig. 1: Comparison of CEA between cases and control group

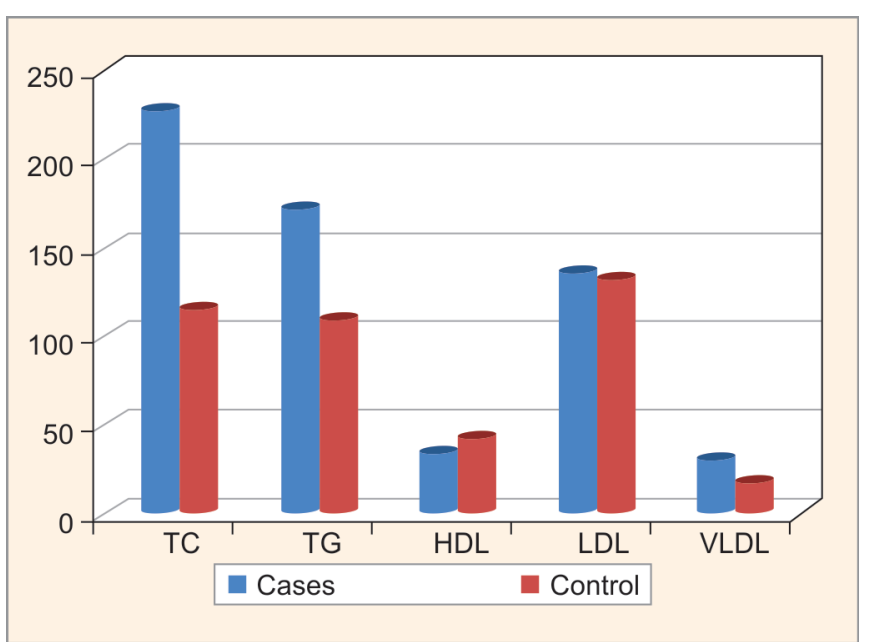

Fig. 2: Comparison of lipid profile levels between cases and control group 
breast cancer patients and the lipid profile is a routine biochemical parameter, which can help to detect the status of malignancy.

\section{REFERENCES}

1. Mealiffe ME, Stokowski RP, Rhees BK, et al. Assessment of clinical validity of a breast cancer risk model combining genetic and clinical information. J Natl Cancer Inst 2010;102(21):1618-1627. DOI: 10.1093/ jnci/djq388.

2. Fitzmaurice C, Akinyemiju TF, Lami FH, et al. Global, regional, and national cancer incidence, mortality, years of life lost, years lived with disability, and disability-adjusted life-years for 29 cancer groups, 1990 to 2016: a systematic analysis for the global burden of disease study. JAMA Oncol 2018;4(11):1553-1568. DOI: 10.1001/jamaoncol.2018.2706.

3. Jemal A, Bray F, Center MM, et al. Global cancer statistics. Cancer J Clin 2011;61(2):69-90. DOI: 10.3322/caac.20107.

4. Bray F, Ferlay J, Soerjomataram I, et al. Global cancer statistics 2018: globocan estimates of incidence and mortality worldwide for 36 cancers in 185 countries. CA Cancer J Clin 2018;68(6):394-424. DOI: 10.3322/caac.21492.

5. Sharma S, Desai PB, Metgudmath RB. Evaluation of serum adenosine Deaminase and retinol in patients with laryngeal cancer. Indian J Pharm Biol Res 2013;1(4):30-34. DOI: 10.30750/ijpbr.1.4.6.

6. Serdarevic N, Mehanovic $\mathrm{S}$. The possible role of tumor antigen CA 15-3, CEA and ferritin in malignant and benign disease. J Health Sci 2012(2):138-143. DOI: 10.17532/jhsci.2012.52.

7. Hoskovec D, Varga J, Konecna E, et al. Levels of CEA and ca 19-9 in the sera and peritoneal cavity in patients with gastric and pancreatic cancers. Acta Cir Bras 2012;27(6):410-416. DOI: 10.1590/S010286502012000600009.

8. Shao $Y$, Sun $X, \mathrm{He} Y$, et al. Elevated levels of serum tumor markers CEA and CA15-3 are prognostic parameters for different molecular subtypes of breast cancer. PLoS One 2015;10(7):e0133830. DOI: 10.1371/journal.pone.0133830.

9. Fakih MG, Padmanabhan A.CEA monitoring in colorectal cancer.what you should know. Oncology (Williston Park) 2006;20(6):579-588. , 594, 596 passim.

10. Laisupasin $\mathrm{P}$, Thompat W, Sukarayodhin S, et al. Comparison of serum lipid profiles between normal controls and breast cancer patients. J Lab Physicians 2013;5(1):38-41. DOI: 10.4103/0974-2727.115934.

11. Boyle $P$, Boniol $M$, Koechlin $A$, et al. Diabetes and breast cancer risk: a meta-analysis. Br J Cancer 2012;107(9):1608-1617. DOI: 10.1038/ bjc.2012.414.

12. Rakha EA, Sayed ME, Green AR, et al. Prognostic markers in triplenegative breast cancer. Cancer 2007;109(1):25-32. DOI: 10.1002/ cncr.22381.

13. Wang WS, Lin JK, Lin TC, et al. Plasma von Willebrand factor level as a prognostic indicator of patients with metastatic colorectal carcinoma. World J Gastroenterol 2005;11(14):2166-2170. DOI: 10.3748/wjg.v11.i14.2166.

14. Faridi SH, Khan MA, Siddiqui B, et al. Role of serum Carcinoembryonic antigen (CEA) as a tumor marker in breast cancer. Int J Adv Med Health Res 2014;1(2):57-60. DOI: 10.4103/2349-4220.148002.

15. Li X, Dai D, Chen B, et al. Clinicopathological and prognostic significance of cancer antigen 15-3 and Carcinoembryonic antigen in breast cancer: a meta-analysis including 12,993 patients. Dis Markers 2018;2018:9863092. DOI: 10.1155/2018/9863092.

16. Bhat S A, MirM R, Majid S, et al. Serum lipid profile of breast cancer patients in Kashmir. J Investig Biochem 2013;2(1):26-31. DOI: 10.5455/ jib.20121125075314.

17. Abdelsalam KEA, Hassan IK, Sadig IA. The role of developing breast cancer in alteration of serum lipid profile. J Res Med Sci 2012;17(6):562-565. 